\title{
Nicotine's Effects on Attentional Efficiency in Alcoholics
}

\author{
Sara Jo Nixon, Andrea Lawton-Craddock, Rick Tivis, and Natalie Ceballos
}

\begin{abstract}
Background: Historically, the concomitant use of nicotine among alcoholics has not been methodologically accounted for. Given the observed cognitive enhancing effects of acute nicotine on attentional processes, it is important that the potentially positive effects of nicotine be disentangled from the negative effects of chronic alcohol dependence. The current study was conducted to address this question and to test the hypothesis that alcoholics who are regular smokers are more sensitive to the effects of nicotine on cognition as compared to regular smoking community controls.

Methods: A 2 [drug group; alcoholics $(n=28)$, community controls $(n=27)$ ] X 2 nicotine dose level [low (7 mg dose) vs. high (14 or $21 \mathrm{mg}$ dose)] double-blind design was used to assess the differential effects of nicotine dose on a battery of neurocognitive tests focusing on attentional efficiency.

Results: As expected, the alcoholic group performed more poorly than did the control group. However, of greater interest to the current study was the finding that alcoholic participants differentially benefited from nicotine administration, as demonstrated in the differential dose effect.

Conclusion: The concomitant use of nicotine may serve to "mask" or "overcome" some of the negative effects of chronic alcohol dependence in newly recovering alcoholics. This potential effect has significant implications for treatment development and further understanding of the process of recovery of function in chronic alcoholics.
\end{abstract}

Key Words: Neurocognition, Acute Nicotine, Alcoholism, Cognitive Efficiency.

$\mathrm{D}$ ECREMENTS OF COGNITIVE functioning have long been observed among detoxified alcohol-dependent participants in the early stages of recovery. Standardized neuropsychological tests and computerized cognitive batteries have revealed mild to moderate impairment in problem solving and abstraction, visual-spatial ability, perceptual-motor function, attention, cognitive flexibility, and memory (Nixon, 1999; Parsons and Nixon, 1993; Sullivan et al., 2000b). These findings are congruent with neuroimaging and pathology reports of alcohol-related changes in both white and gray matter of the brain (Cardenas et al., 2005; Harper et al., 2005). In brief, observations of detectable brain changes and detriments in cognitive functioning in recently abstinent alcoholics are relatively robust findings (Evert and Oscar-Berman, 1995; Nixon, 1999; O'Neill et al., 2001; Oscar-Berman, et al., 1997; Pfefferbaum et al., 1995; Rourke and Loberg, 1996).

Several models have been offered to account for diverse neuropsychological decrements exhibited by chronic alcoholics. One approach is to focus on the underlying cognitive

From the Department of Psychiatry, McKnight Brain Institute, Newell, Gainesville, Florida (SJN); Lynn Health Science Institute, Oklahoma City, Oklahoma (ALC); Statistically Speaking LLP, Meridian, Idaho (RT); and Department of Psychology, Texas State University, San Marcos, Texas (NC).

Received for publication June 6, 2006; accepted September 4, 2007.

Reprint request: Sara Jo Nixon, PhD, Department of Psychiatry, McKnight Brain Institute (SJN), 100 S. Newell, Gainesville, FL 32611; Fax: 352-392-2579; E-mail: sjnixon@ufl.edu

Copyright (C) 2007 by the Research Society on Alcoholism.

DOI: $10.1111 /$ j.1530-0277.2007.00526.x mechanisms involved. The component-process model, proposed by Nixon and Parsons (1991), has examined various cognitive processes that may be adversely affected by habitual excessive alcohol exposure (Nixon, 1993, 1999; Parsons and Nixon, 1993). Of particular interest is the concept of cognitive efficiency. Efficiency is typically regarded in terms of speed/accuracy tradeoffs (Sternberg, 1984); however, it may also be more broadly conceptualized as the ability to attend to accurate, relevant information while ignoring inaccurate, irrelevant information within a defined time frame (Nixon, 1999).

The concept of cognitive efficiency in substance abusing populations has been examined in a variety of contexts using neuropsychological assessments, computerized neurocognitive batteries, event-related encephalographic potentials, and even postural stability (Nixon and Parsons, 1991; Nixon et al., 1998, 2002; Sullivan et al., 2000a,b). The application of cognitive efficiency most relevant to the current study involves the use of efficiency ratios (accuracy divided by mean reaction time) obtained in response to a computerized neurocognitive battery. Glenn and Parsons $(1990,1992)$ used such a battery to assess problem solving, memory, visual-spatial function, perceptual-motor speed, and verbal comprehension in detoxified alcoholic men and women. Results indicated that alcoholic participants were less efficient when compared to their control counterparts. These findings have been extended in various alcoholic and polysubstance abusing subgroups (Lawton-Craddock et al., 2003; Nixon et al., 1998).

However, the previously mentioned studies of cognitive efficiency did not systematically address the potential 
confound of simultaneous nicotine use. The cognitive effects of nicotine are particularly relevant given the substantial numbers of smokers among alcohol-dependent individuals seeking treatment. Epidemiological studies have shown that smoking prevalence ranges from 80 to $95 \%$ in alcohol abusers (Le, 2002; Miller, 1999; Patten et al., 1996). The association between nicotine and enhanced cognitive performance has been extensively researched (Foulds et al., 1996; Hughes, 1991; Murphy and Klein, 1998; Rusted et al., 1997; Witte et al., 1997). One of the most consistent findings in human studies is that nicotine administration facilitates attentional and working memory processes as evidenced by its improvement of cognitive performance in adults with attention-deficit/hyperactivity (Cogar et al., 1996; Conners et al., 1996; Levin et al., 1996) and Alzheimer's disease (Jones et al., 1992; Murray and Abeles, 2002; Newhouse et al., 1988; Sahakian and Coull, 1994; White and Levin, 1999).

Nicotine's potential as a cognitive enhancer is an especially important consideration in the study of cognitive performance in alcoholic populations, as attentional and short-term memory deficits are often observed this group. Thus, based upon previous research in nonsubstance abusing groups, it is logical to suggest that alcohol-related impairments of attention and short-term memory could be diminished with nicotine exposure. Further, it is of particular interest to ascertain if nicotine administration differentially impacts performance among alcoholic participants relative to community controls.

Recent work by Nixon and colleagues (Ceballos et al., $2005,2006)$ reports the predicted differential enhancement of cognitive performance in substance abusing groups (alcoholics, illicit stimulant abusers, and those who abuse both alcohol and illicit stimulants) following acute nicotine administration. However, there is an obvious need for replication of this work and to refine the locus of this effect. Thus, the overarching objective of this study was to address the question of whether nicotine administration would improve cognitive efficiency deficits in alcoholic participants. More specifically, we focused on efficiency in attention because nicotine is known to enhance attentional processes (e.g., Levin et al., 2006).

Furthermore, we anticipated that deficits in attentional efficiency are posited to underlie much of the cognitive impairment observed in detoxified alcoholics. Working from these assumptions, we predicted that detoxified smoking alcoholics (a group that is historically known to exhibit cognitive impairments), would benefit to a greater degree than would smoking community controls. Because we were not examining the effects of withdrawal, we anticipated that this effect would be observed through a differential (better) response to the higher dose of nicotine.

To that end, participants in both groups were randomly assigned to receive either a high or low dose transdermal nicotine patch prior to performing a computerized cognitive efficiency battery. Based on the current literature, it was hypothesized that alcoholics exposed to higher levels of nicotine would exhibit greater cognitive efficiency on tasks relying heavily on attentional processes than would those alcoholics exposed to lower nicotine levels. By contrast, such pronounced differences in neurocognitive performance between nicotine dose groups were not expected to emerge among smoking controls. That is, we predicted that alcoholics would demonstrate greater sensitivity to the dose manipulation than would community controls.

\section{MATERIALS AND METHODS}

\section{Participants}

The current study was approved by the Institutional Review Board of the University of Oklahoma Health Sciences Center and informed consent was obtained prior to each phase of the study, including screening, diagnostic interview and neurocognitive testing.

In an effort to clarify the neurocognitive effects of acute nicotine administration in alcohol-dependent individuals and community controls, a 2 (drug group) X 2 (nicotine dose level) experimental design was implemented. Participants were randomly assigned to either a low (7 mg) or high (21 mg for men; $14 \mathrm{mg}$ for women) dose of the transdermal nicotine patch (Nicoderm CQ). Participants ranged in age from 21 to 59. This age range was chosen to reduce the effects of brain development in adolescents and cognitive decline among elderly participants, issues that would be expected to impact the results of the neurocognitive tests employed in the current study. Participants had 9 to 18 years of education, and smoked a minimum of 10 cigarettes per day during the course of the previous year. Those participants selected for participation provided written informed consent and were reimbursed for their time and travel costs.

Twenty-eight abstinent alcohol-dependent individuals $(21 \mathrm{men} / 7$ women) were enlisted from inpatient and outpatient drug treatment centers in Oklahoma City and the surrounding communities. Participants recruited for the alcohol group were required to meet DSM-IV (American Psychiatric Association, 1994) guidelines for alcohol dependence, as reflected by the NIMH Diagnostic Interview Schedule (DIS-IV; Robins et al., 1995). Furthermore, a QuantityFrequency Index (QFI; Cahalan et al., 1969) was computed for each participant to determine the average absolute ethanol intake per day (in ounces) for the 6-month period prior to being tested. There was no difference between men and women alcoholics on reported QFI $(t(26)=0.41, p>0.28)$. Men alcoholics reported an average daily intake of $11.34 \pm 6.15$ ounces of absolute ethanol, whereas women alcoholics reported an average daily intake of $10.21 \pm 6.47$ ounces of absolute ethanol (approximately 18 to 20 standard drinks per day). All alcoholic subjects had achieved between 21 and 75 days of abstinence at the time of laboratory testing. Every attempt was made to recruit alcoholics without a substantial history of other drug use. In the final sample of alcoholics, $7 \%$ (2 subjects) reported use of multiple (polydrug) substances in addition to alcohol; 25\% (7 subjects) reported the concurrent use of marijuana and alcohol with or without other substances; and $18 \%$ (5 subjects) reported use of stimulants in addition to alcohol.

Community controls $(n=27 ; 20$ men $/ 7$ women) were enlisted from Oklahoma City and neighboring communities were recruited via newspaper advertisements and word-of-mouth. As with the alcohol-dependent participants, a QFI was calculated for controls, and their substance use history was recorded. Participants with current or lifetime diagnosis of alcohol dependence were excluded and QFI's were $\leq 1.9$ (less than an average of $\sim 2$ drinks per day). Previous or current occasional marijuana use was also allowed. However, individuals were questioned to ensure that use did not occur within the 48 hours prior to testing. Because studies have indicated that prior marijuana use does not result in gross cognitive impairments, these 
individuals were not eliminated from subsequent analyses (Pope et al., 1995; Solowij, 1998). Control participants reported no abuse of illicit substances within the 10 years prior to testing.

The racial/ethnic composition did not differ significantly across groups $\left(\chi^{2}(1)<0.5, p>0.4\right)$. The control group $(n=21)$ was $78 \%$ Caucasian, and the ALC group $(n=24)$ was $86 \%$ Caucasian. As a result of inadequate representations of ethnic minorities, more explicit analyses were not performed.

\section{Screening}

Participants were required to complete three screening phases prior to study participation. The first consisted of a phone screen given to all potential control participants who called our laboratory inquiring about participation. This phone screen obtained information concerning age, education, general health status, and a brief history of alcohol, drug, and nicotine usage.

If individuals remained eligible after the initial screen, they were asked to complete a screening packet. Alcoholic participants generally were administered this screening packet in their treatment facility. The screening packet gathered demographic information and included the Shipley Institute of Living Scale (SILS) Vocabulary subtest (Zachary, 1986) to exclude those with a remedial vocabulary level (i.e., < a score of 20; Lezak, 1995). Additionally, participants completed the Spielberger State Anxiety Inventory (SSAI) (Spielberger, 1983), and the Beck Depression Inventory (BDI-II) (Beck et al., 1996). Also contained in the questionnaire was an extensive review of past and current alcohol, drug, and nicotine use including questions concerning chronicity, frequency, and typical usage patterns. Air expired carbon monoxide (CO) reading was also obtained from this phase of the screening process for use as a comparison measure in the verification of smoking abstinence on the day of laboratory testing.

Medical information was obtained to exclude those with neurological disorders, substantial head trauma (i.e., repeated or prolonged periods of unconsciousness), or serious medical illnesses (e.g., diabetes, cancer, heart, or liver condition). In addition, individuals were excluded if they were currently pregnant or had any disorder, such as chronic skin conditions or hypertension, which would contraindicate the use of the nicotine patch. For this study, hypertension was defined as a blood pressure reading greater than or equal to 140/90, in accordance with guidelines issued by the National Institutes of Health (United States Department of Health and Human Services, 2006). Medical charts located in the treatment center confirmed the information given by alcoholics. For controls, responses to the initial medical interview were substantiated by a self-report medical questionnaire given to all participants at the time of testing. In addition, physicians reviewed all medical information prior to the participant being tested to insure health requirements were met.

Individuals who continued to meet entry criteria following the first two stages of screening were given a psychiatric assessment using the NIMH Diagnostic Interview Schedule (DIS-IV) (Robins et al., 1995) by a trained research assistant to exclude those individuals who endorsed a lifetime diagnosis of any psychotic disorder (e.g., any of the schizophrenic disorders or a psychotic depression), or bipolar disorder or current major depressive disorder. They were also provided a detailed description of the study including information regarding nicotine administration procedures and urine screening that would test for other drugs of abuse and pregnancy (for women).

\section{Neurocognitive Materials}

As part of a more comprehensive test battery that took approximately five hours to complete, participants were administered the following tests: a simple reaction time test (time to respond to the presentation of a stimulus), the Sternberg test (Sternberg, 1966, 1975), the Digit Symbol Test (Forms A \& B; Wechsler, 1981; Beatty et al., 2000), the Stroop test (Stroop, 1935; Trennery et al., 1989), and the Trail Making Test (Forms A \& B; Reitan, 1955; Reitan \& Wolfson, 1985).

\section{Sternberg Task (Sternberg, 1966, 1975)}

On this computerized task of short-term memory, participants were shown digits within sets, varying in size from one to six digits in length. After the final digit of the set was shown, a visual cue (a plus sign) was presented followed by a probe digit. After indicating the presence or absence of the probe digit among the set of digits, the participant recalled the digit set by typing the corresponding numbers on the keyboard. The dependent variables were percent correct and mean reaction time.

\section{Digit Symbol Test (Wechsler, 1981)}

On Form A, the participant was instructed to quickly fill in the empty squares with the symbol that corresponded to the number. A total of 90 seconds was allotted for completion. The test requires the participant to attend to both the number and the symbol and the shift attention between these sets of stimuli. On Form B, (adapted for use in this laboratory, see Beatty et al., 2000) the participant was instructed to quickly fill in the empty spaces with the symbol that corresponded to the number plus one for 90 seconds. The number of squares accurately completed represented the score.

\section{Stroop Test (Stroop, 1935; Trennery et al., 1989)}

This classical test of interference consisted of three different trials. On the first trial (i.e., Stroop Word Trial), the participant was given a sheet containing names of colors printed in black ink and was required to read as many of these words aloud as possible in a 45-second time frame. The second trial, referred to as the Stroop Color Trial, required the participant to name the color of the ink used to print a series of "X"s. The X's were randomly presented in blue, green, or red ink. The third trial, called the Stroop Color-Word Trial, had a list of color words (e.g., "green," "blue," etc.) printed in an ink color that was different from the name of the color word (e.g., the word "blue" printed in red ink). The participant was asked to name the color of the ink and to avoid saying the word that spelledout a conflicting color. Scoring was based on the number of colors correctly identified within a 45-second time limit.

\section{Trail Making Test (Trails A and Trails B, Reitan, 1955; Reitan and Wolfson, 1993)}

On Trails A, the participant was instructed to draw lines to connect numbered circles in a consecutive manner (e.g., 1 to 2,2 to 3 , etc.). On Trails B, the participant was required to draw lines connecting numbered circles to lettered circles in a consecutive fashion. Participants were urged to perform the task as quickly as possible. Scores were based on the time required to correctly connect the circles. Trails A requires directed attention to over-learned number sequences. Trails B requires that participants attend to the order and shifting between two sets of over-learned sequences (numbers 1 to 13 and alphabet letters $\mathrm{A}-\mathrm{L}$.

\section{Nicotine Administration}

Prior to participation, participants were instructed to abstain from smoking for a minimum of five hours prior to testing (e.g., 2 to $7 \mathrm{AM}$. or overnight) in order to attain optimal nicotine effects. After arriving at the laboratory and obtaining written consent and a urine specimen, the participant submitted a $\mathrm{CO}$ reading to ensure that minimal nicotine deprivation had occurred (i.e., the $\mathrm{CO}$ value obtained from day of testing was $50 \%$ lower than the baseline value taken during 
screening phases or was $12 \mathrm{ppm}$ or lower). Blood pressure was also measured to exclude individuals with clinically significant hypertensive readings. A urine sample was collected and screened for drugs of abuse. Women participants also underwent a urine test for pregnancy. A breath sample was obtained from all participants to ensure abstinence from alcohol.

Participants were then randomly assigned to either the "low dose" or "high dose" condition (e.g., low dose patch $\{7 \mathrm{mg}\}$ or high dose patch $\{14 \mathrm{mg} / 21 \mathrm{mg}\})$ using a double-blind controlled design. Patches were positioned on the upper back left shoulder of each participant. The neurocognitive testing began approximately 3.5 hours after patch administration. Because we were not interested in acute withdrawal, no placebo manipulation was included. We understand that this prevents the assessment of a main effect of nicotine (i.e., nicotine as compared to no drug) per se. However, our primary objective was to observe a differential effect of nicotine in the drugwithdrawn alcoholic group versus the community control group, as illustrated in the differential dose effect in this group.

A standard cigarette delivers approximately $1 \mathrm{mg}$ of nicotine via inhalation; thus, the application of a $21 \mathrm{mg}$ transdermal nicotine patch is designed to deliver a slow, steady dose of nicotine that is roughly equivalent to smoking 21 cigarettes over a 16 to 24 hour period (GlaxoSmithKline, 2006). Given the differential pharmacokinetics of the nicotine patch versus cigarette smoking, we did, however, assess deprivation and withdrawal symptoms. In order to measure the potential level of withdrawal in these participants, the Withdrawal Symptoms Checklist (WSC) (adapted from Hughes and Hatsukami, 1986) was given to participants before patch attachment and throughout the testing session in 1-hour intervals. In addition, the BDI-II (Beck et al., 1996), and Spielberger State Anxiety Inventory (SSAI) (Spielberger, 1983) were re-administered to monitor changes in affective symptomatology. At the conclusion of the test, all participants were debriefed on which dose of patch they received and instructed to refrain from smoking for 5 hours following patch removal. In addition, they received cessation information from the American Cancer Society.

\section{Data Analysis}

A 2 (drug group) $\times 2$ (nicotine dose) experimental design was analyzed with an ANOVA to determine the effect of nicotine dose, group affiliation, and their interaction on overall efficiency. Because of inadequate representation of women among the various groups, statistical procedures addressing the effect of gender could not be performed. It should be noted that the pattern of the mean comparisons was similar when only men were included although power was reduced. Given the focus of this study, the primary statistical test was the $t$-test performed either to examine group differences (between alcoholics and controls) or dose differences (within alcoholics and within controls). Where the test for unequal variance was significant the Satterthwaite adjusted results were used. The data were analyzed using SAS Version 9 statistical package.

\section{RESULTS}

Various indices of nicotine usage patterns were examined (see Table 1). There were no significant differences between the ALC group and the control group on number of cigarettes smoked daily $[t(51)=0.81, p>0.42]$. Likewise, these groups also did not differ in their chronicity (in years) of cigarette smoking $[t(50)=0.35, p>0.72]$. In addition, there were no significant differences between the groups on the $\mathrm{CO}$ values submitted during the screen $[t(53)=1.43, p>0.15]$ or the CO levels read at the time of testing $[t(53)=0.62$,
Table 1. Nicotine Usage Patterns by Control and Alcoholics Groups: Means (SD, range)

\begin{tabular}{lcc}
\hline & Controls $(n=27)$ & Alcoholics $(n=28)$ \\
\hline \# of Cigarettes/day & $21.2(8.3,10-40)$ & $23.4(11.1,10-60)$ \\
Smoking chronicity (years) & $16.6(3.7,9-26)$ & $17.0(5.1,10-33)$ \\
CO $^{\mathrm{a}}$ at screen & $19.6(8.3,9-38)$ & $23.7(12.2,8-53)$ \\
$\mathrm{CO}^{\mathrm{a}}$ at testing & $10.3(6.5,3-35)$ & $11.4(6.3,2-26)$ \\
$\mathrm{SCQ}^{\mathrm{b}}$ total & $49.1(13.7,15.2-72.5)$ & $48.5(9.9,31.4-70.7)$ \\
WSC $^{\mathrm{c}}$ & $4.2(3.1,0-16)$ & $6.1(4.3,0-17)$ \\
FTND $^{d}$ total $^{*}$ & $3.8(2.3,0-9)$ & $6.0(2.3,2-10)$ \\
\hline
\end{tabular}

${ }^{a}$ Carbon monoxide measured in ppm., ${ }^{\mathrm{b}}$ Smoking consequences questionnaire (Copeland et al., 1995)., 'Withdrawal symptoms check-

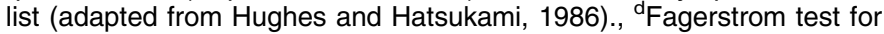
nicotine dependence (Heatherton et al., 1991)., ${ }^{*}$ Significant group differences $(p<0.09)$.

$p>0.53]$ with each group demonstrating approximately a $50 \%$ decrease from the former to the latter value. Importantly, the subgroups [group (alc/control) X dose (high/low)] also did not differ in the number of hours between last cigarette and the administration of the transdermal patch (means ranged from 8.4 to 9.8 hours: $F<1$ ).

The number of consequences associated with tobacco use was also similar across alcoholic and control groups as measured by the Smoking Consequences Questionnaire (SCQ; Copeland et al., 1995), [t(47) $=0.18, p>0.85]$, as well as endorsement of withdrawal symptoms (WSC) preceding task administration $[\operatorname{WSC}(t(53)=1.87, p>0.06)]$.

Given concern that differential withdrawal symptoms (WSC scores) might influence performance, dose effects were also examined. This analysis revealed a significant patch effect $[t(53)=2.43,(t(53)=2.36, p<0.02) p=0.02]$ with the high dose condition reporting more withdrawal symptoms than the low dose condition $[5.3 \pm 3.6(5.25$ (high) vs. $3.3 \pm 2.43 .25$ (low)]. There were no significant correlations between WSC scores and performance measures when the entire sample was considered or when the controls were considered alone. When only alcoholics were analyzed, none of the correlations achieved significance after Bonferroni corrections were made. However, there were two performance measures which produced provocative correlations with the WSC: the Sternberg $[r(n=28)=0.39, p=0.04]$ and the Stroop Color Words (interference trial) $[r(n=28)=0.45 p=$ $0.02]$. Given the weak nature of these findings, additional analyses were not conducted, however, potential implications for these unexpected findings are noted in the discussion section.

There was disparity between the groups on the Fagerstrom Test for Nicotine Dependence measure (FTND) (Heatherton et al., 1991). The ALC group had a significantly higher score on this instrument than controls $[t(47)=3.42, p \leq 0.002]$. Cutoff scores of 4 or higher have been used by the FTND to designate nicotine dependence (Breslau and Johnson, 2000). The percentage of individuals endorsing a score of 4 or higher in each of these groups was: controls (12; 44\%), ALC (19, 68\%). 
Table 2. Demographic and Self-Report Measures of Control and Alcoholic Groups: Means (SD, range)

\begin{tabular}{lcc}
\hline & $\begin{array}{c}\text { Controls }(n=27), \\
(n=8)\end{array}$ & $\begin{array}{c}\text { Alcoholics }(n=28), \\
(n=8)\end{array}$ \\
\hline Age & $33.6(10.1,21-54)$ & $38.4(7.9,22-53)$ \\
Years of education* & $13.1(1.0,12-15)$ & $12.3(1.9,9-18)$ \\
BDI-II $^{2 *}$ & $3.4(4.7,0-16)$ & $12.6(9.9,0-35)$ \\
SSAI $^{b}$ & $45.0(6.8,34-59)$ & $49.4(10.3,35-76)$ \\
SILS-V $^{c *}$ & $17.7(1.5,14.7-20.2)$ & $16.6(2.1,13.5-20.2)$ \\
QFI $^{d *}$ & $.44(0.56,0-1.9) 0-1.87$ & $11.1(6.1,2.0-28.2)$ \\
\hline
\end{tabular}

aBeck Depression Inventory II (Beck et al., 1996)., 'b Spielberger State Anxiety Inventory (Spielberger, 1983)., " Shipley Institute of Living-Vocabulary Subscale (Zachary, 1986)., 'Quantity Frequency Index (Cahalan et al., 1969)., ${ }^{*}$ Significant group differences $(p<0.05)$.

The proportion of participants reporting such levels of nicotine dependence did not differ significantly between the groups $\left[\chi^{2}(1)=3.06, p>0.08\right]$. In addition, correlation analyses within groups revealed that FTND scores were not associated with performance $(r s<.31, p>0.15)$.

Table 2 illustrates findings of the demographic and selfreport measures among controls and alcoholics. There were no significant differences between the groups on age $[t(53)=1.98, p>0.05]$. A statistically significant difference was detected between the groups on years of education $[t(40.5)=2.18, p \leq 0.04]$ with the ALC group reporting fewer years of education than controls. Although this difference is statistically significant, it is doubtful that the difference in 12 and 13 years of education is clinically significant.

Significant differences were also observed on the SILS-V (vocabulary) subtest. Analyses revealed that the control group achieved significantly higher scores than the ALC group $[t(53)=2.20, p \leq 0.04]$. There were no significant differences between the groups on reported anxiety levels as assessed by the SSAI measure, although it approached significance $[t(53)=1.87, p>0.06]$. On the BDI-II scale, the ALC group endorsed significantly greater depressive symptomatology, as compared with the control group $[t(37.4)=4.37$, $p \leq 0.0001]$. As expected, there were significant differences between the groups on QFI with the ALC group endorsing higher levels of ethanol consumption than controls $[t(27.5)=9.12, p \leq 0.0001]$. When a Bonferroni correction was applied to this set of analyses, only the BDI-II and QFI differences achieved significance.

Correlational analyses were performed for each of the groups and across such groups to ascertain if age, education, BDI-II, SSAI, and SILS-V were significantly related to any of the neurocognitive tasks. Findings from these correlational analyses indicated no consistent associations among the variables for any of the groups. For the BDI-II, the only significant correlations were for the substance abusers (attentional efficiency, $r(n=26)=0.40, p<0.04$ and Stroop Color Words, $r(n=27)=0.41, p<0.04)$. None of the other measures correlated with BDI-II $(|r| \mathrm{s}<0.37, p$ s $>0.06)$. Also none of the measures correlated with BDI-II for the controls $(|r| \mathrm{s}<0.29, p \mathrm{~s}>0.15)$. In the controls, the SSAI and the Trails B was significantly correlated $[r(n=25)=$ $-0.57, p<0.003]$. However, this correlation did not achieve significance for the substance abusers $[r(n=27)=-.22$, $p>0.27]$. None of the other measures were correlated with the SSAI $(|r| \mathrm{s}<0.31, p>0.15)$ for either group. The SILS-V was not associated with any of the neurocognitive task for the controls $(|r| \mathrm{s}<0.31, p \mathrm{~s}>12)$. There was an association between the SILS-V and the Stroop words $[r(n=$ 28) $=0.42, p<0.03]$ and the Trails $\mathrm{B}[r(n=27)=0.50, p$ $<0.008)$ for the substance abusers. When a Bonferroni correction was applied to these analyses, only the association between the SILS-V and the Trails B for the substance abusers retained significance. In an attempt to further reduce the potential impact of relevant demographic variables, we also subjected the overall attentional efficiency measure and the individual subtests to ANCOVA controlling for age, SSAI and BDI-II. Importantly, the results were not changed. Therefore, the results and means presented are unadjusted means.

To evaluate the effectiveness of the random assignment of patch, demographic variables were analyzed to detect performance differences within the groups. None of the differences achieved significance [ $t \mathrm{~s}<1.2, p \mathrm{~s}>0.24]$. The means by subgroup are presented in Tables 3 and 4.

\section{Analyses of Neurocognitive Measures}

An efficiency ratio was derived for each of the tasks by dividing percent correct by mean reaction time for each participant. For the Digit Symbol (D/S) test (Forms A \& B) and the three Stroop trial tasks, the time element of the efficiency ratio was fixed (i.e., 90 seconds and 45 seconds, respectively) in adherence to standardized administration procedures. An overall attentional efficiency index was calculated by averaging the efficiency ratios from the four tasks.

Consistent with expectations, a significant main effect for drug group was observed $[F(1,48)=5.59, p<0.03]$ with controls achieving a significantly higher overall efficiency

Table 3. Demographic and Self-Report Measures Within Control Groups

\begin{tabular}{|c|c|c|c|c|}
\hline & \multicolumn{4}{|c|}{ Controls } \\
\hline & \multicolumn{2}{|c|}{ Low patch $(n=14)$} & \multicolumn{2}{|c|}{ High patch $(n=13)$} \\
\hline & Mean (SD) & Range & Mean (SD) & Range \\
\hline Age & $35.7(9.5)$ & $25-54$ & $31.2(10.6)$ & $21-51$ \\
\hline Years of education & $13.0(0.96)$ & $12-15$ & $13.3(1.0)$ & $12-15$ \\
\hline $\mathrm{BDI}-\|^{\mathrm{a}}$ & $1.9(3.5)$ & $0-13$ & $5.1(5.4)$ & $0-16$ \\
\hline SSAI $^{b}$ & $40.6(4.2)$ & $34-47$ & 43.3 (7.3) & $35-54$ \\
\hline SILS-V' & $18.1(1.5)$ & $16.2-20.2$ & $17.2(1.5)$ & $14.7-19.8$ \\
\hline $\mathrm{QFI}^{\mathrm{d}}$ & $0.3(0.38)$ & $0-1.18$ & $0.5(0.71)$ & $0.1-1.9$ \\
\hline FTND $^{\mathrm{e}}$ & $3.1(1.7)$ & $0-6$ & $4.5(2.6)$ & $1-9$ \\
\hline $\begin{array}{l}\text { Average number } \\
\text { of cigarettes smoked }\end{array}$ & $20.0(7.9)$ & $10-40$ & $22.5(8.9)$ & $10-35$ \\
\hline
\end{tabular}

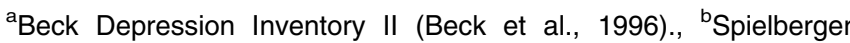
State Anxiety Inventory (Spielberger, 1983)., "'Shipley Institute of Living-Vocabulary Subscale (Zachary, 1986)., d'Quantity Frequency Index

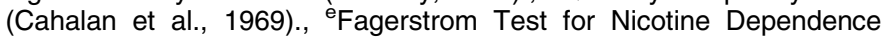
(Heatherton et al., 1991). 
Table 4. Demographic and Self-Report Measures Within Alcoholic Groups

\begin{tabular}{|c|c|c|c|c|}
\hline & \multicolumn{4}{|c|}{ Alcoholics } \\
\hline & \multicolumn{2}{|c|}{ Low patch $(n=13)$} & \multicolumn{2}{|c|}{ High patch $(n=15)$} \\
\hline & Mean (SD) & Range & Mean (SD) & Range \\
\hline Age & $36.2(8.4)$ & $22-49$ & $40.3(7.3)$ & $32-53$ \\
\hline Years of education & $11.9(1.4)$ & $9-15$ & 12.5 (2.3) & $9-18$ \\
\hline BDI-II & $12.8(8.9)$ & $0-31$ & $12.5(11.0)$ & $1-35$ \\
\hline $\mathrm{SSAl}^{\mathrm{b}}$ & 48.7 (8.4) & $37-61$ & $51.4(11.2)$ & $34-76$ \\
\hline SILS-V ${ }^{\mathrm{C}}$ & $16.0(1.8)$ & $13.5-19.8$ & $17.1(2.2)$ & 13.9-20.2 \\
\hline $\mathrm{QFI}^{\mathrm{d}}$ & $10.5(6.11)$ & $2.0-22.8$ & $11.6(6.3)$ & 6.1-28.2 \\
\hline $\begin{array}{l}\text { Years with an } \\
\text { alcohol problem }\end{array}$ & $14.2(7.0)$ & $2-25$ & $17.1(7.9)$ & 5-30 \\
\hline FTND $^{\mathrm{e}}$ & $5.8(2.7)$ & 2-9 & $6.1(2.1)$ & $2-10$ \\
\hline $\begin{array}{l}\text { Average number } \\
\text { of cigarettes smoked }\end{array}$ & $23.3(9.2)$ & $10-40$ & $23.5(12.9)$ & $10-60$ \\
\hline
\end{tabular}

a Beck Depression Inventory II (Beck et al., 1996)., ' Spielberger State Anxiety Inventory (Spielberger, 1983)., 'Shipley Institute of Living-Vocabulary Subscale (Zachary, 1986)., 'Withdrawal symptoms checklist (adapted from Hughes and Hatsukami, 1986)., 'Quantity Frequency Index (Cahalan et al., 1969)., ${ }^{\mathrm{e}}$ Fagerstrom test for nicotine dependence (Heatherton et al., 1991).

score compared to their ALC counterparts. Likewise, a significant main effect for dose was found $[F(1,48)=7.67$, $p<0.009$ ] with the high dose group performing significantly better than the low dose group. The group $\mathrm{x}$ nicotine dose interaction did not achieve significance $[F<1]$.

To focus on the benefit of nicotine administration to persons in treatment for alcohol dependence, as reflected in our primary hypothesis, we examined the effect of nicotine on attentional efficiency measure within the alcoholic group. The dose effect was significant $[t(25)=1.99, p \leq 0.03]$. To further investigate this effect, we examined the individual measures that make up the attentional efficiency measure. Given the directionality of the hypothesis a one tailed $t$-test was used. The results are reported in Table 5. Findings from these analyses revealed that alcoholic individuals given the high dose performed better on the Sternberg task than those receiving the low dose $[p \leq 0.03]$. Likewise, significant differences were obtained in the Stroop Word Trial, [ $p \leq 0.03]$, the Stroop Color Words (the interference trial) $[p<0.04]$ and the Trails $\mathrm{B}[p \leq 0.05]$ with individuals in high dose cells performing better than those in low dose cells. There was also a trend for significance on the Trails A $[p \leq 0.06]$ The control subjects

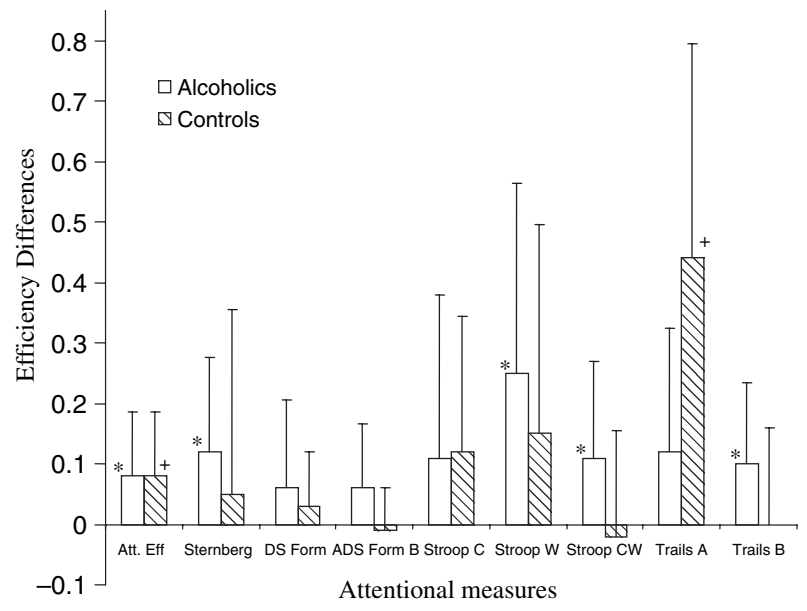

Fig. 1. Efficiency improvement on attentional measures for alcoholics and controls. Efficiency differences were derived by subtracting the efficiency ratios obtained by the low-dose group from that obtained by the high-dose group for alcoholic and control groups, separately. AffEff, overall attentional efficiency; DS, digit symbol A and B; Stroop C, color naming; Stroop W, word naming; Stroop $\mathrm{CW}$, interference trial. ${ }^{*}$ Differences in alcoholics at $p<0.05 ;+$ differences in community controls at $p<0.05$.

showed a significant dose effect on the overall attentional efficiency index $[t(23)=1.93, p \leq 0.04]$ However, the pattern was not observed across subtests with the only individual task to show a significant difference being the Trails A $[t(23)=3.14, p \leq 0.003]$ Fig. 1.

\section{DISCUSSION}

As expected, alcoholic participants demonstrated inferior performance on an overall performance index assessing efficiency in sustained attention and short-term memory abilities. Variables, such as age, educational levels, depressive and anxiety symptomatology, and related measures could not explain these observed deficits.

Also consistent with the existing literature were data indicating nicotine's positive affect on attentional and working memory processes. Both animal and human studies indicate that nicotine use enhances attention, learning, and working memory skills (Foulds et al., 1996; Levin et al., 1996; Mirza \& Stolerman, 1998; Rusted et al., 1997). Current research using treatment of nicotine agonists to ameliorate symptoms on

Table 5. Differences in Attentional Measures Within the Alcoholics

\begin{tabular}{|c|c|c|c|c|}
\hline Task & $\begin{array}{l}\text { Low patch }(n=13) \text {, } \\
\text { mean }(S D \text {, range) }\end{array}$ & $\begin{array}{l}\text { High patch }(n=15) \\
\text { mean }(S D \text {, range) }\end{array}$ & $t(\mathrm{DF})$ & $P$ \\
\hline Overall attentional efficiency & $0.47(0.10,0.27-0.61)$ & $0.55(0.11,0.39-0.79)$ & $1.99(25)$ & 0.03 \\
\hline Sternberg & $0.42(0.16,0.16-0.73)$ & $0.54(0.15,0.34-0.83)$ & $2.05(26)$ & 0.03 \\
\hline Digit symbol Form A & $0.55(0.16,0.24-0.80)$ & $0.61(0.13,0.43-0.81)$ & $1.07(26)$ & 0.15 \\
\hline Digit symbol Form B & $0.45(0.11,0.20-0.61)$ & $0.51(0.10,0.39-0.71)$ & $1.23(26)$ & 0.12 \\
\hline Stroop color & $1.43(0.23,1.04-1.84)$ & $1.54(0.31,0.98-2.22)$ & $1.06(26)$ & 0.15 \\
\hline Stroop words & $1.98(0.30,1.56-2.49)$ & $2.22(0.33,1.60-2.84)$ & $2.03(26)$ & 0.03 \\
\hline Stroop color words & $0.76(0.14,0.47-0.98)$ & $0.87(0.18,0.67-1.42)$ & $1.88(26)$ & 0.04 \\
\hline Trails A & $0.71(0.24,0.25-1.04)$ & $0.83(0.17,0.47-1.09)$ & $1.62(26)$ & 0.06 \\
\hline Trails B & $0.25(0.11,0.11-0.50)$ & $0.35(0.16,0.13-0.60)$ & $1.76(25)$ & 0.05 \\
\hline
\end{tabular}


populations known to be afflicted with attentional and working memory deficits, such as individuals with attention deficit hyperactivity disorder and Alzheimer's disease, illustrate nicotine's ability to improve such cognitive functions (Cogar et al., 1996; Conners et al., 1996; Jones et al., 1992; Levin et al., 1996; Newhouse et al., 1988; Sahakian and Coull, 1994). These findings are also supported by electrophysiological and neuroimaging studies, which show that nicotine reduces P3 latency and increases blood perfusion in areas responsible for visual attention (Edwards et al., 1985; Ghatan et al., 1998; LeHouezec et al., 1994; McNamara et al., 1990). The current work, considering the differential effects of low and high nicotine doses, corroborates these conclusions. Results revealed a significant main effect for dose on the overall performance index of efficiency with the high dose group attaining a significantly higher score on this measure than their low dose counterparts.

Of particular interest to this inquiry were analyses (directed by the working hypothesis) conducted on nicotine dose differences within each drug group. Consistent with our hypothesis, alcoholic individuals in the high patch condition performed better than those in the low patch condition across the subtests compromising the attentional efficiency measure. While a significant effect was observed in the controls, only one of the subtests demonstrated the effect. That is, nicotine dose comparisons within drug group revealed that nicotine exposure had a more consistent effect on alcohol-dependent individuals as compared to controls.

This latter observation has significance in both the research and clinical arenas. In the research arena, neuropsychological studies conducted thus far on alcoholic populations may be underestimating cognitive deficits in these samples because of the possible counteractive effects of acute nicotine administration. In the clinical arena, attempts by treatment providers to stop smoking along with alcohol misuse in substance abuse populations may ultimately have adverse effects on certain aspects of treatment adherence and occupational functioning. For example, alcoholics quitting smoking without an adequate pharmacological substitution may have impaired ability to process information and diminished capacity to sustain attention in group therapy sessions even if longer term outcomes are not necessarily compromised (for discussion see Gulliver et al., 2000; Hughes et al., 2003; Prochaska et al., 2004), Given these important issues, treatment providers who implement a program advocating the cessation of smoking and alcohol abuse concurrently may consider administering nicotine replacement therapy (e.g., transdermal nicotine \{patch\}, nicotine polacrilex \{gum\}, nasal spray), at least for some period of time. These nicotine replacement medications may produce the desirable cognitive changes (previously provided by cigarettes) until complete detoxification occurs and observed deficits are reversed or coping skills are developed to deal with more chronic difficulties with concentration and short-term memory.

Because there has been a growing interest in treating alcoholism and tobacco dependence concurrently, further studies are needed to ascertain the specific cognitive deficits that may arise from this approach and which individuals are most vulnerable to having these problems. By understanding these important issues, professionals can implement creative and practical treatment strategies. Given the complexity associated with having dual dependence of alcohol and nicotine, an integrated pharmacological, psychological, and behavioral intervention would be optimal in treating this population.

\section{Limitations}

Inadequate numbers of women and minorities limit the present study's ability to infer its results to these populations. Further, the age range of the current study prohibits generalization of these findings to other groups, such as adolescents or adults older than 59 years of age. The potential effects of nicotine on substance abusers in these age groups remains to be addressed in future research. Additionally, despite matching on many nicotine-related indices and other self-report measures, there were significant differences between alcoholics and controls on some variables. Although these variables were found not to impact the pattern of performance obtained on neuropsychological tasks, future studies should attempt to match controls and alcohol-dependent individuals on a host of variables including affective state, smoking history (chronicity and number typically smoked) as well as age, education and gender. Additionally, if possible, groups might be examined for differences in pattern of smoking as well as early exposure to second-hand smoke (both during childhood and in utero). Analyses regarding some of these issues are now on-going in our laboratory.

Furthermore, it may be argued that a more optimal design would incorporate a nonsmoking group that would be represented across drug and nicotine dose cells. This more complete design, however, has inherent methodological problems. Specifically, these include (a) ethical concerns of exposing nonsmokers to nicotine, (b) lower functional tolerance of nonsmokers to nicotine's side effects (e.g., nausea and lightheadedness), and (c) difficulties recruiting sufficient numbers of nonsmoking alcoholics given the high smoking rates in this population. The current design although limited is directly responsive to the primary question and provides provocative data for further study.

Finally, although we attempted to avoid confounds associated with withdrawal from nicotine, We recognize that the application of the $7 \mathrm{mg}$ patch may not eliminate all withdrawal symptoms. However, this confound seems unlikely in the current study given data indicating that the $7 \mathrm{mg}$ dose group did not report higher withdrawal symptoms than those receiving the higher dose. Interestingly, the high dose group reported more rather than less withdrawal symptoms. This finding was counterintuitive. One possibility we considered, in light of the weak but provocative associations between WSC and performance for the alcoholic group, is that the higher dose produces greater activation or arousal which, in turn, results in endorsing experiences consistent with withdrawal 
symptoms at least in treatment seeking alcoholics who are undergoing dual drug abstinence (alcohol and temporary nicotine). That is, the WSC may have served as a proxy for arousal rather than withdrawal, per se, under the current study conditions. Additional work is needed to examine this possibility further.

\section{Overview}

Although preliminary, these data reinforce current interest in nicotine's role in the concomitant use of other substances. The current study suggests that nicotine's acute cognitive enhancing effects may be influenced by the user's drug history. The study also, indirectly, draws attention to the distinction between drug effects and outcomes more likely associated with mode of administration. That is, it illustrates the importance of distinguishing between the direct effects of the addictive substance nicotine and the indirect effects more likely because of its typical mode of administration, i.e., cigarette smoking. In the current work, the acute effects of nicotine administered via transdermal patch for alcoholics appear to be positive. However, studies of chronic smokers demonstrate changes in brain structure and function associated with poorer cognitive functioning (Durazzo et al., 2007; Meyerhoff et al., 2006). Systematic studies disentangling acute from chronic effects and the effects of drug (i.e., nicotine) versus those associated with route of administration (i.e., smoking) are needed.

\section{ACKNOWLEDGMENTS}

This project was supported by a grant from NIAAA (RO1 AA09163 to S.J. Nixon, P.I.), and through a training grant \#5T32AA0722 stipend (F. Holloway, P.I., deceased) and OUHSC Department of Psychiatry mini-grant awarded to A. Lawton-Craddock. This research was completed in partial fulfillment of the requirements for the Doctor of Philosophy degree in Biological Psychology (A. Lawton-Craddock). A preliminary report of the results was presented at the annual meeting of the Research Society on Alcoholism, and the abstract appeared in Alcoholism: Clinical \& Experimental Research (2003, supplement no. 5, p. 140A). Special thanks to Robert A. Prather for his help in the structure of the final manuscript.

\section{REFERENCES}

American Psychiatric Association (1994) Diagnostic and Statistical Manual of Mental Disorders. 4th ed. APA, Washington DC.

Beatty WW, Tivis R, Stott HD, Nixon SJ, Parsons OA (2000) Neuropsychological deficits in sober alcoholics: Influences on chronicity and recent alcohol consumption. Alcohol Clin Exp Res 24:149-154.

Beck AT, Steer RA, Brown GK (1996) Beck Depression Inventory. 2nd ed. The Psychological Corporation, San Antonio.

Breslau N, Johnson E (2000) Predicting smoking cessation and major depression in nicotine-dependent smokers. Am J Public Health 90:1122-1127.

Cahalan D, Cissin L, Crossley H (1969) American Drinking Practices: A National Study of Drinking Behaviors and Attitudes Monograph No. 6. Rutgers Center of Alcohol Studies, New Brunswick.
Cardenas VA, Studholme C, Meyerhoff DJ, Song E, Weiner MW (2005) Chronic active heavy drinking and family history of problem drinking modulate Regional brain tissue volumes. Psychiatry Res 138:115-130.

Ceballos NA, Tivis R, Lawton-Craddock A, Nixon SJ (2005) Visual-spatial attention in alcoholics and illicit stimulant abusers: effects of nicotine replacement. Prog Neuropsychopharmacol Biol Psychiatry 29:97-107.

Ceballos NA, Tivis R, Lawton-Craddock A, Nixon SJ (2006) Nicotine and cognitive efficiency in alcoholics and illicit stimulant abusers: implications of smoking cessation for substance users in treatment. Subst Use Misuse 41:265-281.

Cogar RW, Moe KL, Serafetinides EA (1996) Attention deficit disorder in adults and nicotine dependence: psychobiological factors in resistant to recovery? J Psychoactive Drugs 28:229-240.

Conners CK, Levin ED, Sparrow E, Hinton SC, Ernhardt D, Meck WH, Rose JE, March J (1996) Nicotine and attention in adult attention deficit hyperactivity disorder (ADHD). Psychopharmacol Bull 32:67-73.

Copeland AL, Brandon TH, Quinn EP (1995) The smoking consequences questionnaire-adult: measurement of smoking outcome expectancies of experienced smokers. Psychol Assess 7:484-494.

Durazzo TC, Rothlind JC, Gazdzinski S, Banys P, Meyerhoff DJ (2007) Chronic smoking is associated with differential neurocognitive recovery in abstinent alcoholic patients: A preliminary investigation. Alcohol Exp Clin Res 31:1114-1127.

Edwards JA, Wesnes K, Warburton DM, Gale A (1985) Evidence of more rapid stimulus evaluation following cigarette smoking. Addict Behav 10:113-126.

Evert DL, Oscar-Berman M (1995) Alcohol-related cognitive impairments: an overview of how alcoholism may affect the workings of the brain. Alcohol Health Res World 19:89-96.

Foulds J, Stapleton J, Swettenham J, Bell N, McSorley K, Mah R (1996) Cognitive performance effects of subcutaneous nicotine in smokers and never-smokers. Psychopharmacology 127:31-38.

Ghatan PH, Ingvar M, Erikson L, Stone-Elanders S, Serrander M, Ekberg K, Wahren J (1998) Cerebral effects of nicotine during cognition in smokers and non-smokers. Psychopharmacology 136:179-190.

GlaxoSmithKline (2006) Clear NicodermCQ: Frequently asked questions. GlaxoSmithKline, Middlesex, UK. http://www.nicodermcq.com/faqs.aspx\#07. Accessed July 24, 2006.

Glenn SW, Parsons OA (1990) The role of time in neuropsychological performance: investigation and application in alcoholic populations. Clin Neuropsychol 4:344-354.

Glenn SW, Parsons OA (1992) Neuropsychological efficiency measures in male and female alcoholics. J Stud Alcohol 53:546-552.

Gulliver SB, Kalman D, Rohsenow DJ, Colby SM, Eaton CA, Monti PM (2000) Smoking and drinking among alcoholics in treatment: cross-sectional and longitudinal relationships. J Stud Alcohol 61:157-163.

Harper C, Matsumoto I, Pfefferbaum A, Adalsteinsson E, Sullivan EV, Lewohl J, Dodd P, Taylor M, Fein G, Landman B (2005) The pathophysiology of 'brain shrinkage' in alcoholics - structureal and molecular changes and clinical implications. Alcohol Clin Exp Res 29:1106-1115.

Heatherton TF, Kozlowski LT, Frecker RC, Fagerstrom KO (1991) The Fagerstrom test for nicotine dependence: a revision of the Fagerstrom tolerance questionnaire. Br J Addict 86:1119-1127.

Hughes JR (1991) Distinguishing withdrawal relief and direct effects of smoking. Psychopharmacology 104:409-410.

Hughes JR, Hatsukami D (1986) Signs and symptoms of tobacco withdrawal. Arch Gen Psychiatry 43:289-294.

Hughes JR, Novy P, Hatsukami DK, Jensen J, Callas PW (2003) Efficacy of nicotine patch in smokers with a history of alcoholism. Alcohol Clin Exp Res 27:946-954.

Jones GMM, Sahakian BJ, Levy R, Warburton DM, Gray JA (1992) Effects of acute subcutaneous nicotine on attention, information processing and short-term memory in Alzheimer's disease. Psychopharmacology 108:485494.

Lawton-Craddock A (2003) Nicotine's effects on neurocognitive performance in alcoholics. Unpublished doctoral dissertation. University of Oklahoma Health Sciences Center, Oklahoma City, Oklahoma. 
Lawton-Craddock A, Nixon SJ, Tivis RJ (2003) Cognitive efficiency in stimulant abusers with and without alcohol dependence. Alcohol Clin Exp Res $27: 457-464$

Le AD (2002) Effects of nicotine on alcohol consumption. Alcohol Clin Exp Res 26:1915-1916.

LeHouezec J, Halliday R, Benowitz NL, Callaway E, Naylor H, Herzig K (1994) A low dose of subcutaneous nicotine improves information processing in non-smokers. Psychopharmacology 114:628-634.

Levin ED, Conners CK, Sparrow E, Hinton S, Meck W, Rose JE, Ernhardt D, March J (1996) Nicotine effects on adults with attention-deficit/hyperactivity disorder. Psychopharmacology 123:55-63.

Levin ED, McClernon FJ, Rezvani AJ (2006) Nicotinic effects on cognitive function: behavioral characterization, pharmacological specification, and anatomic localization. Psychopharmacology, 184:523-539.

Lezak MD (1995) Neuropsychological Assessment 3rd ed. Oxford University Press, New York.

McNamara D, Larson DM, Rapoport SI, Soncrant TT (1990) Preferential metabolic activation of subcortical brain areas by acute administration of nicotine in rats. J Cereb Blood Flow Metab 10:48-56.

Meyerhoff DJ, Tizabi Y, Staley JK, Durazzo TC, Glass JM, Nixon SJ (2006) Smoking comorbidity in alcoholism: neurobiological and neurocognitive consequences. Alcohol Clin Exp Res 30:253-264.

Miller NS (1999) Mortality risks in alcoholism and effects of abstinence and addiction treatment. Psychiatr Clin North Am 22:371-383.

Mirza NR, Stolerman ID (2000) The role of nicotinic and muscarinic acetylcholine receptors in attention. Psychopharmacology 148:243-250.

Murphy FC, Klein RM (1998) The effects of nicotine on spatial and nonspatial expectancies in covert orienting task. Neuropsychologia 36:1113-1114.

Murray KN, Abeles N (2002) Nicotine's effects on neural and cognitive functioning in an aging population. Aging Ment Health 6:129-138.

Newhouse PA, Sunderland T, Tariot PN, Blumhardt CL, Wingartner H, Mellow A, Murphy DL (1988) Intravenous nicotine in Alzheimer's disease: a pilot study. Psychopharmacology 95:171-175.

Nixon SJ (1993) Application of theoretical models to the study of alcohol-induced brain damage, in Alcohol-Induced Brain Damage Research Monograph, Number 22 (Hunt WA, Nixon SJ eds), pp 213 228. National Institute on Alcohol Abuse and Alcoholism, Rockville MD.

Nixon SJ (1999) Neurocognitive performance in alcoholics: Is polysubstance abuse important? Psychol Sci 10:181-185.

Nixon SJ, Parsons OA (1991) Alcohol-related efficiency deficits using an ecologically valid test. Alcohol Clin Exp Res 20:21-24.

Nixon SJ, Paul R, Phillips M (1998) Cognitive efficiency in alcoholics and polysubstance abusers. Alcohol Clin Exp Res, 27:1414-1420.

Nixon SJ, Tivis R, Ceballos N, Varner JL, Rohrbaugh J (2002) Neurophysiological efficiency in male and female alcoholics. Prog Neuropsychopharmacol Biol Psychiatry 26:919-927.

Oscar-Berman M, Shagrin B, Evert DL, Epstein C (1997) Impairments of brain and behavior: The neurological effects of alcohol. Alcohol Health Res World 21:65-75

O'Neill J, Cardenas VA, Meyerhoff DJ (2001) Effects of abstinence on the brain: quantitative magnetic resonance imaging and magnetic resonance spectroscopic imaging in chronic alcohol abuse. Alcohol Clin Exp Res 25:1673-1682.

Parsons OA, Nixon SJ (1993) Neurobehavioral sequelae of alcoholism. BehavNeurol 11:205-218.

Patten CA, Martin JE, Owen N (1996) Can psychiatric and chemical dependency treatment units be smoke free? J Subst Abuse Treat 13:107-118.

Pfefferbaum A, Sullivan EV, Mathalon DH, Shear PK, Rosenbloom MJ, Lim KO (1995) Longitudinal changes in magnetic resonance imaging in brain volumes in abstinent and relapsed alcoholics. Alcoholism Clin Exp Res 19:1177-1191.
Pope HG, Gruber AJ, Yugelun-Todd D (1995) The residual neuropsychological effects of cannabis: the current status of research. Drug Alcohol Dep 38:25-34

Prochaska JJ, Delucchi K, Hall SM (2004) A meta-analysis of smoking cessation interventions with individuals in substance abuse treatment or recovery. J Consult Clin Psychol 72:1144-1156.

Reitan RM (1955) The relation of the trail making test to organic brain damage. J Consulting Psychol 19:393-394.

Reitan RM, Wolfson D (1985) The Halstead-Reitan Neuropsychological Test Battery: Theory and clinical interpretation. Neuropsychology Press, Tucson.

Reitan RM, Wolfson D (1993) The Halstead-Reitan Neuropsychological Test Battery: Theory and Clinical Interpretation. Neuropsychology Press, Tucson, AZ.

Robins LN, Cottler L, Bucholz K, Compton W (1995) The Diagnostic Interview Schedule, Version IV. Washington University, St. Louis.

Rourke SB, Loberg T (1996) Neurobehavioral correlates of alcoholism, in Neuropsychological Assessment of Neuropsychiatric Disorders (Grant I, Adams KM eds), pp 423-485. Oxford University Press, New York.

Rusted JM, Graupner L, Tennant A, Warburton DM (1997) Effortful processing is a requirement for nicotine-induced improvements in memory. Psychopharmacology 138:362-368.

Sahakian BJ, Coull JT (1994) Nicotine and tetrahydroaminoacradine: evidence for improved attention in patients with dementias of the Alzheimer type. Drug Dev Res 31:80-88.

Solowij N (1998) Cannabis and Cognitive Functioning. Cambridge University Press, Cambridge, England.

Spielberger CD (1983) Manual for the State-Trait Anxiety Inventory. Consulting Psychologists Press, Palo Alto.

Sternberg S (1966) High-speed scanning in human memory. Science 153:652654.

Sternberg S (1975) Memory scanning: new findings and current controversies. Q J Exp Psychol A 27:1-32.

Sternberg RJ (1984) Toward a triarchic theory of human intelligence. Behav Brain Sci 7:269-315.

Stroop JR (1935) Studies of interference in serial verbal reactions. J Exp Psychol 18:643-662.

Sullivan EV, Rosenbloom MJ, Lim KO, Pfefferbaum A (2000a) Longitudinal changes in cognition, gait, and balance in abstinent and relapsed alcoholic men: Relationships to changes in brain structure. Neuropsychology 14:178 188.

Sullivan EV, Rosenbloom MJ, Pfefferbaum A (2000b) Pattern of motor and cognitive deficits in detoxified alcoholic men. Alcohol Clin Exp Res 24:611621.

Trennery MR, Crosson B, DeBoe J, Leber WR (1989) Stroop Neuropsychological Screening Test Manual. Psychological Assessment Resources, Odessa, FL.

United States Department of Health and Human Services (2006) What is high blood pressure? U.S. Department of Health and Human Services, Washington, DC. http://www.nhlbi.nih.gov/health/dci/Diseases/Hbp/ HBP WhatIs.html. Accessed July 24, 2006.

Wechsler D (1981) WAIS-R Manual. The Psychological Corporation, New York.

White HK, Levin ED (1999) Four-week nicotine skin patch treatment effects on cognitive performance in Alzheimer's disease. Psychopharmacology 143:158-165.

Witte EA, Davidson MC, Marocco RT (1997) Effects of altering brain cholinergic activity on covert orienting of attention: comparison of monkey and human performance. Psychopharmacology 132:324-334.

Zachary RA (1986) Shipley Institute of Living Scale, Revised Manual. Western Psychological Services, Los Angeles. 